\title{
A SYMPLECTIC FIXED POINT THEOREM FOR COMPLEX PROJECTIVE SPACES
}

\author{
BY BARRY FORTUNE AND ALAN WEINSTEIN ${ }^{1}$
}

1. Arnold's conjecture. An automorphism $\psi$ of a symplectic manifold $(P, \omega)$ is homologous to the identity if there is a smooth family $\psi_{t}(t \in[0,1])$ of automorphisms such that the time-dependent vector field $\xi_{t}$ defined by $d \psi_{t} / d t=\xi_{t} \circ \psi_{t}$ is globally hamiltonian; i.e. if there is a smooth family $H_{t}$ of real-valued functions on $P$ such that $\left.\xi_{t}\right\rfloor \omega=d H_{t}$. It was conjectured by Arnold [1], as an extension of the Poincaré-Birkhoff annulus theorem $[\mathbf{3}, \mathbf{7}]$, that every automorphism of a compact symplectic manifold $P$, homologous to the identity, has at least as many fixed points as a function on $P$ has critical points.

Arnold's conjecture was proven by Conley and Zehnder [4] for the torus $T^{2 n} \approx \mathbf{R}^{2 n} / \mathbf{Z}^{2 n}$ with its usual symplectic structure. They show that every symplectic automorphism of $T^{2 n}$, homologous to the identity, has at least $n+1$ fixed points, and at least $2^{2 n}$ if all are nondegenerate. Their method was extended in [8] to prove a version of Arnold's conjecture for arbitrary $P$ under the additional assumption that the hamiltonian vector field $\xi_{t}$ is sufficiently $C^{0}$ small.

In this note we announce a proof of Arnold's conjecture for the complex projective space $\mathbf{C} P^{n}$ with its standard symplectic structure. We prove that a symplectic diffeomorphism of $\mathbf{C} P^{n}$, homologous to the identity, has at least $n+1$ distinct fixed points. (By the Lefschetz fixed point theorem, any continuous map from $\mathbf{C} P^{n}$ to itself, homotopic to the identity, has at least $n+1$ fixed points counted with multiplicities.) For $n=1\left(\mathbf{C} P^{1} \approx S^{2}\right)$ the result was already known [1], but with a proof which worked only in this two-dimensional case.

The proof for $T^{2 n}$ in [4] made use of a variational principle in which the fixed points of the map were identified with periodic solutions of a timedependent hamiltonian system and then identified with critical points of a functional on the space of contractible loops on $T^{2 n}$. The corresponding functional in the case of $\mathbf{C} P^{n}$ is multiple valued, and there are other difficulties connected with the curved geometry of $\mathbf{C} P^{n}$, so we need a new approach. Our trick is to consider the hamiltonian system on $\mathbf{C} P^{n}$ as the reduction, in the sense of [6], of a hamiltonian system on $\mathbf{C}^{n+1}$ and then adapt recently developed methods [2] for finding periodic orbits in $\mathbf{C}^{n+1}$. This method is similar to that of Conley and Zehnder in that a problem on a compact manifold is lifted to a problem on euclidean space invariant under a group of transformations.

Received by the editors January 26, 1984.

1980 Mathematics Subject Classification. Primary 58F05.

${ }^{1}$ Research partially supported by NSF Grant MCS 80-23356. 
2. Lifting to $\mathbf{C}^{n+1}$. Consider $\mathbf{C}^{n+1}$ with its usual symplectic structure $\operatorname{Im} \sum d z_{\imath} \wedge d \bar{z}_{\imath}$. The hamiltonian $K(z)=\sum z_{\imath} \bar{z}_{\imath}$ generates the periodic flow $T_{\mu}\left(z_{1}, \ldots, z_{n+1}\right)=\left(e^{2 \imath \mu} z_{1}, \ldots, e^{2 \imath \mu} z_{n}\right)$ with period $\pi$, and hence an action of $S^{1}=\mathbf{R} / \pi \mathbf{Z}$ (the Hopf fibration). The reduced manifold $K^{-1}(1) / S^{1}$ can be identified with $\mathbf{C} P^{n}$, and any $S^{1}$-invariant hamiltonian system on $\mathbf{C}^{n+1}$ induces a system on $\mathbf{C} P^{n}$, called the reduced system. Our idea is to use this procedure in the opposite direction.

Fixed points of $\psi: \mathbf{C} P^{n} \rightarrow \mathbf{C} P^{n}$ are the same as solution curves $\tilde{\sigma}:[0,1] \rightarrow$ $\mathrm{C} P^{n}$ with $\tilde{\sigma}(0)=\tilde{\sigma}(1)$ for the time-dependent hamiltonian system which generates the family $\psi_{t}$ connecting the identity to $\psi$. Let $\tilde{H}_{t}$ be the hamiltonian family for this system; since each $\tilde{H}_{t}$ contains an arbitrary constant, we may assume that $\tilde{H}_{t}(x)>0$ for all $t$ in $[0,1]$ and all $x$ in $\mathbf{C} P^{n}$. Now let $H_{t}: \mathbf{C}^{n+1} \rightarrow \mathbf{R}$ be the unique function which is homogeneous of degree 2 and whose restriction to $K^{-1}(1)=S^{2 n+1}$ is the pullback of $\tilde{H}_{t}$. Then $H_{t}$ is $S^{1}$ invariant and defines a time-dependent hamiltonian system on $\mathbf{C}^{n+1}$ whose reduced system is $H_{t}$.

By the general theory of reduction, we know that $S^{2 n+1}$ is an invariant manifold for $H_{t}$, and the orbits of $\tilde{H}_{t}$ on $\mathbf{C} P^{n}$ are the images of orbits of $H_{t}$ on $S^{2 n+1}$. Furthermore, if $\tilde{\sigma}$ is the image of $\sigma$, then $\tilde{\sigma}(1)=\tilde{\sigma}(0)$ if and only if $\sigma(\mathbf{1})=T_{\mu} \sigma(0)$ for some $\mu$ in $\mathbf{R} / \pi \mathbf{Z}$. If we change the hamiltonian $H_{t}$ to $H_{t}+\lambda K$ for some $\lambda \in \mathbf{R}$, then the "flow" of $H_{t}+\lambda K$ will still project to that of $\tilde{H}_{t}$, but now by choosing $\lambda(\bmod \pi)=\mu$ we can make $\sigma(1)=\sigma(0)$. In other words, to each closed solution curve $\tilde{\sigma}$ for $\tilde{H}_{t}$ and, hence, to each fixed point of $\psi$ there corresponds a collection of pairs $(\sigma, \lambda)$ where $\lambda \in \mathbf{R}$ and $\sigma$ is a closed solution curve for $H_{t}+\lambda K$ on $S^{2 n+1}$. The set of all pairs $(\sigma, \lambda)$ corresponding to a given fixed point is diffeomorphic to $S^{1} \times \mathbf{Z}$.

By Hamilton's principle the closed solution curves for $H_{t}+\lambda K$ on $\mathbf{C}^{n+1}$ are exactly the critical points of the functional

$$
\begin{aligned}
g(z) & =\int_{0}^{1}-i\left(z^{\prime}(t), z(t)\right) d t+\int_{0}^{1} H_{t}(z(t)) d t+\lambda \int_{0}^{1}|z(t)|^{2} d t \\
& =A(z)+H(z)+\lambda K(z) .
\end{aligned}
$$

Since we are interested in critical points for all possible values of $\lambda$, we may consider $\lambda$ as a Lagrange multiplier and look for critical points of $f(z)=$ $A(z)+H(z)$ constrained to the infinite-dimensional sphere $K^{-1}(1)$.

We are thus faced with two problems. The first is to do the analysis which shows that $f(z)$ has many critical points on $K^{-1}(1)$, and the second is to show that all these critical points cannot belong to fewer than $n+1$ families of type $S^{1} \times \mathbf{Z}$ coming from distinct fixed points of $\psi$.

3. Critical point analysis. The solution of the problems stated at the end of $\S 2$ forms the content of [5] and will only be summarized briefly here.

It turns out that the critical point theory developed in $[\mathbf{2}]$, based on the notion of relative index, is applicable to our problem, with some modifications made to permit working on the sphere $K^{-1}(1)$ within the space of loops of Sobolev class $H^{1 / 2}$ in $\mathbf{C}^{n+1}$. The values of the Lagrange multiplier $\lambda$ are then found to be equal to the critical values of the functional $f$ on $K^{-1}(1)$. 
The minimax nature of the critical point theory makes it possible to estimate these values by comparison with the action functional $A$. A combinatorial argument then shows that these critical values cannot lie in less than $n+1$ cosets of $\mathbf{R}(\bmod \pi \mathbf{Z})$ unless some critical values merge, in which case $\psi$ would have uncountably many fixed points.

\section{REFERENCES}

1. V. I. Arnold, Mathematical methods of classical mechanics, Springer-Verlag, New York, 1978.

2. H. Berestycki, J.-M. Lasry, G. Mancini and B. Ruf, Existence of multiple periodrc orbuts on star-shaped hamiltonian surfaces, preprint, 1983.

3. G. D. Birkhoff, Proof of Poincaré's geometric theorem, Trans. Amer. Math. Soc. 14 (1913), 14-22.

4. C. C. Conley and E. Zehnder, The Birkhoff-Lewis fixed point theorem and a conjecture of V. I. Arnold, Invent. Math. 73 (1983), 33-49.

5. B. Fortune, A symplectic fixed-point theorem for $\mathbf{C} P^{n}$, Ph.D. thesis, Univ. of Cal. at Berkeley, 1984.

6. J. Marsden and A. Weinstein, Reduction of symplectic manifolds with symmetry, Rep. Math. Phys. 5 (1974), 121-130.

7. H. Poincaré, Sur un théorème de géométrie, Rend. Circ. Mat. Palermo 33 (1912), 375 407.

8. A. Weinstein, $C^{0}$ perturbation theorems for symplectic fuxed points and lagrangian intersectrons, Séminaire sud-rhodanien de géométrie III, Travaux en Cours, Hermann, (1984). (Another version will appear in the proceedings of the 1983 AMS Summer Institute on Nonlinear Functional Analysis and Applications, Proc. Sympos. Pure Math., Amer. Math. Soc., Providence, R.I.)

Department of Mathematics, University of California, Berkeley, CALIFORNIA 94720 